\title{
BMJ Open QUIT EMR trial: a prospective, observational, multicentre study to evaluate quality and 24 hours post- transport morbidity of interhospital transportation of critically ill patients: study protocol
}

\author{
Ulrich Strauch, ${ }^{1}$ Dennis C J J Bergmans, ${ }^{1}$ Joachim Habers, ${ }^{2}$ Jochen Jansen, ${ }^{3}$ \\ Bjorn Winkens, ${ }^{4}$ Dirk J Veldman, ${ }^{5}$ Paul M H J Roekaerts, ${ }^{1}$ Stefan K Beckers ${ }^{6}$
}

To cite: Strauch U, Bergmans DCJJ, Habers J, et al. QUIT EMR trial: a prospective, observational, multicentre study to evaluate quality and 24 hours posttransport morbidity of interhospital transportation of critically ill patients: study protocol. BMJ Open 2017:7: e012861. doi:10.1136/ bmjopen-2016-012861

- Prepublication history and additional material is available. To view please visit the journal (http://dx.doi.org/ 10.1136/bmjopen-2016012861).

Received 27 May 2016 Revised 16 January 2017 Accepted 1 February 2017

CrossMark

For numbered affiliations see end of article.

Correspondence to Dr Ulrich Strauch; u.strauch@mumc.nl

\section{ABSTRACT}

Introduction: It is widely accepted that transportation of critically ill patients is high risk. Unfortunately, however, there are currently no evidence-based criteria with which to determine the quality of various interhospital transport systems and their impact on the outcomes for patients. We aim to rectify this by assessing 2 scores which were developed in our hospital in a prospective, observational study. Primarily, we will be examining the Quality of interhospital critical care transportation in the Euregion Meuse-Rhine (QUIT EMR) score, which focuses on the quality of the transport system, and secondarily the SEMROS (Simplified EMR outcome score) which detects changes in the patient's clinical condition in the 24 hours following their transportation.

Methods and analysis: A web-based application will be used to document around 150 pretransport, intratransport and post-transport items of each patient case.

To be included, patients must be at least 18-years of age and should have been supervised by a physician during an interhospital transport which was started in the study region.

The quality of the QUIT EMR score will be assessed by comparing 3 predefined levels of transport facilities: the high, medium and low standards. Subsequently, SEMROS will be used to determine the effect of transport quality on the morbidity 24 hours after transportation.

It is estimated that there will be roughly 3000 appropriate cases suitable for inclusion in this study per year. Cases shall be collected from 1 April 2015 until 31 December 2017.

Ethics and dissemination: This trial was approved by the Ethics committees of the university hospitals of Maastricht (Netherlands) and Aachen (Germany). The study results will be published in a peer reviewed journal. Results of this study will determine if a prospective randomised trial involving patients of various categories being randomly assigned to different levels of transportation system shall be conducted.

\section{Strengths and limitations of this study}

Uniform web-based registration of critically ill patient transport cases creates a unique database to be used in the assessment of two newly developed scoring systems which will be introduced in research and clinical practice.

- Outcomes of this prospective study will provide an international multicentre focused evaluation of a clinically relevant quality monitoring score.

- With the use of a recently evaluated scoring system, this study will provide insight into the effects of different modes of transport on patient mortality 24 hours following transportation.

- Voluntary registration of transport data provided by transportation teams means that the possibility of registration bias cannot be excluded.

- Potential registration bias may be intensified by occasional unavailability of follow-up data.

Trial registration number: NTR4937.

\section{INTRODUCTION}

It is widely accepted that transportation of critically ill patients is high risk, resulting in a significant rate of adverse events. ${ }^{1}$ Patient safety can be compromised particularly during interhospital transportation as a result of a lack of sophisticated resuscitation equipment or absence of sufficiently qualified staff. $^{2-10}$ Within the group of patients requiring interhospital transportation, there are two subcategories: those necessitating urgent lifesaving intervention at an expert centre, and those who are dependent on 
Table 1 Definitions of different levels of ground transport systems

Minimum requirements of ambulance and equipment

System A MICU/ITW*

(high)

System B

(medium)

System C

(low)
Minimum requirements of first Minimum requirements of team member

Intensivist†

ICU physicianף

Physician second team member

\section{ICU nurse}

IC paramedicł

Paramedic

Paramedic

*High volume ambulance equipped with: a boarding ramp, ICU ventilation equipment as well as standard ambulance equipment, a minimum of six infusion pumps, invasive monitoring equipment, the ability to reach the patient from all sides, the ability to transport patients with additional medical devices (such as ECMO, NO, IABP), back-up systems for a ventilator/monitoring/defibrillation unit/suction unit and at least $6000 \mathrm{~L}$ of oxygen (or $6000 \mathrm{~L}$ of pressurised oxygen, if required by the particular ventilator system). The unit must also have a stand-alone capacity of at least 120 min.

†Board certified Intensivist.

$\ddagger$ Paramedic with additional intensive care qualification.

$\S$ Standard ambulance equipped with: a standard ambulance equipment, an ICU transport ventilator, a minimum of four infusion pumps, invasive monitoring equipment and $2000 \mathrm{~L}$ of oxygen. The unit must also have a stand-alone capacity of at least $60 \mathrm{~min}$.

IFCCS or a similarly trained physician with at least 6 months intensive care experience.

ECMO, extracorporeal membrane oxygenation; FCCS, fundamental critical care support; IABP, intra-aortic balloon pump; IC, intensive care; ICU, intensive care unit; ITW, Intensivtransportwagen; MICU, Mobile Intensive Care Units; NO, nitric oxide.

continuous intensive care unit (ICU) therapy, including the use of extracorporeal devices. In daily practice in Germany and the Netherlands, there are multiple varieties of ground ambulances available for use in transporting critically ill patients. These include standard ambulances, ICU ambulances and Mobile Intensive Care Units/Intensivtransportwagen (MICU/ITW). Transportation teams usually include a paramedic and a physician, and teams working within an MICU/ITW often include a physician and nurse trained and experienced with ICU therapy. Typically, the dispatch centre coordinates the transportation and the type of mode used is often based on the urgency and severity of the patient's illness.

Non-stop provision of interhospital transport demands a large amount of resources; however, it has been observed that regional cooperation and support has been useful in making this more manageable. A group within the Euregion Meuse-Rhine (EMR) was formed over the Dutch-German border in order to attempt to develop a plan of cooperation to improve emergency and MICU/ITW transportation. Substantial differences in organisation and legislation regarding interhospital transport in the different countries of the project group were discovered, prompting the group to express the necessity for development of a uniform manner of measuring quality of transport systems. Currently, parameters such as adverse event rate, short-term mortality and changes in Sequential Organ Failure Assessment (SOFA) score before and after transport are used to describe quality of transport systems. ${ }^{11-19}$ To combat difficulties in determination of whether a deterioration of a patient's condition during or immediately after transport is attributable to aspects of the transportation, or due to the natural course of the disease ${ }^{15}$ the project group has initiated the "Quality of interhospital critical care transportation in the Euregion Meuse-Rhine trial (QUIT EMR trial)".

The initial step of the trial was development of 2 scores: the QUIT EMR score which focuses on the quality of the transport system and SEMROS (Simplified EMR outcome score), which detects changes in the patient's clinical condition in the 24 hours following their transportation. Scores can be both systematically and manually calculated.

\section{Objectives}

Primary objective: To assess the QUIT EMR score by means of a prospective multicentre study in which three different transport systems, commonly used within the study region, are compared.

Hypothesis: The QUIT EMR score will be demonstrated as being reliable and accurate, and shall show that there is a difference in number and severity of adverse events between groups of patients transported with high, medium or low standard ground transport systems (table 1).

Secondary Objectives:

A. To assess whether transportation outcome (as determined by the QUIT EMR score) influences 24 hour post-transport morbidity (as determined by SEMROS). Hypothesis: Negative transport outcome will lead to a higher 24 hour post-transportation morbidity.

B. To examine if it is possible to identify and define characteristics which can be used in determination of the necessary transportation variety for a patient.

Hypothesis: It will be possible to identify and define characteristics which can be used in determination of the necessary transportation variety for a patient.

C. To identify predictive outcome parameters concerning 24 hour post-transport mortality.

Hypothesis: Pretransport parameters indicating 24 hour post-transport mortality will be identified and defined. 


\section{METHODS}

Design

"Quality and efficacy of interhospital critical care transportation in the Euregion Meuse-Rhine" is an international, prospective, observational multicentre cohort study. There will be no initiation of interventions, only analysis of anonymous data. The study is open for inclusion from 1 April 2015 until 31 December 2017.

\section{Population/inclusion criteria}

All cases included shall be of patients who are over 18 years of age and who undergo interhospital transportation within the MICU region of Maastricht (Netherlands), district of Aachen (Germany), City of Aachen (Germany) or district of Heinsberg (Germany). Current transportation data suggest that up to 3000 cases of interhospital transportations per year take place under the direct supervision of a physician within the study region.

\section{Study parameters}

Around 150 pretransport, intratransport and posttransport parameters will be scored. Details of these data will be available in the extra file 'web application'. The largest registration categories include:

1. Demographics (patient, equipment, ambulance and transportation team related)

2. - Patient status obtained during the intake call

- Patient status on arrival of the transportation team

- Patient status at the end of the transportation

3. Interventions performed by the transportation team

4. Adverse events

5. Dispatch centre data

6. 24 hours follow-up

\section{QUIT EMR and SEMROS score}

The QUIT EMR Score is a dichotomised scoring system. A score of 1 indicates that there was no necessity for intervention by the transportation team, or that the transport team provided adequate interventions, and a score of 0 indicates that interventions from the transportation team were either insufficient or not performed despite indication. The applied algorithm focuses on changes in physiological parameters, and also combines changes with documented interventions being performed by the transport team. Used data can be found in the additional file 'web application' under part 2.2, part 2.3 and part 3.

The QUIT EMR score has been assessed by means of score calculation of 100 transport charts of the Maastricht University Medical Centre+ (MUMC+) Mobile Intensive Care Unit (MICU). These scores were then subsequently dichotomised to 0 or 1 accordingly.

All transport charts were then also assessed and scored 1 (positive judgement) or 0 (negative judgement) by four specialists from MUMC+ (anaesthesiologists and/or intensivists) experienced in interhospital transport.
These specialist scores were calculated using the following criteria:

- Stable situation during transportation without intervention 1

- Stable situation with adequate intervention 1

- Unstable situation with adequate intervention 1

- Unstable situation or changes in crucial physiological parameters indicating necessary intervention without intervention or with inadequate intervention 0

Specialists were free to define whether a situation was stable or not as well as whether or not intervention was adequate. Finally, the QUIT EMR scores and the specialist scores were compared and an agreement level between $84 \%$ and $92 \%$ was found, as well as an interobserver level of agreement of $85-92 \%$.

The Simplified EMR Outcome Score (SEMROS) is a dichotomised score, where 1 indicates that a patient's status remained unchanged or was improved within 24 hours after transportation, and 0 indicates that a patient's condition deteriorated within the 24 hours following transportation. Data used for calculation of SEMROS is accessible in the web application additional files under parts 2.1 and 6 .

Data used to assess this score were 110 cases of patient transportation towards MUMC+, with the use of an MICU. Of these 110 cases, 90 complete data sets were suitable for calculation of pretransport and posttransport SOFA score ${ }^{20}$ and SEMROS. The SOFA score differs from SEMROS in that it requires multiple laboratory values, which in clinically practice may not always be available, for calculation. Using these 90 cases, an observed level of agreement between the SOFA score and SEMROS of $88.9 \%$ was calculated.

- The following definitions were used regarding the SOFA score: 1 (positive outcome) or 0 (negative outcome)

- SOFA score pretransport lower than SOFA score posttransport 0

- SOFA score pretransport equal to SOFA score posttransport 1

- SOFA score pretransport higher than SOFA score post-transport 1

A web-based application for registration of necessary data has been developed by the center for data and information management of Maastricht University. Specially designed algorithms for automatic calculation of the two scores will be implemented in the study web application.

\section{Data registration}

Web application

A web application has been developed to facilitate data registration. Level 1 users (medical staff present during patient transportation) will perform the initial registration, while follow-up data will be obtained by level 2 users (research staff from the participating organisations). An audit layer of the application will track and store information of all changes. 
Level 1 users

For each case, the physician concerned will document in additional files 1 through 4 of the web application: a standardised set of demographic data, transportation system information, clinical data from the time of the intake call, time of arrival of the transportation team at the patient and at time at the end of transportation, interventions performed by the transportation team, and adverse events. The web application (URL: http:// www.eumic.eu) will be accessible through general username/password combinations. Each participating hospital will receive one unique username/password combination. Alternatively, access will be possible using a global username/password combination for each ambulance, based on the cap codes of the vehicles.

Once a case is finalised, the level 1 user will have the opportunity to request a portable document format file of the documented data. Furthermore, a link will become available for the user to send a comment via email directly to the coordinating investigator or to the technical support staff of MUMC+.

There will be no registration of personal data such as name or date of birth to ensure patient privacy. The unique transport code given by the responsible dispatch centre will be noted.

\section{Level 2 users}

Twenty-four hours after completion of patient transportation, further details will be obtained by level 2 users directly contacting the ICU of the receiving hospital. These users will be able to access and ultimately complete data sets from their area of authorisation in the web-based application with the use of personalised username/password combinations. These users will be unable to alter any data entered previously by level 1 workers.

The procedure for level 2 users will be as follows:

A. Login to the database.

B. Observe the overview of transportation cases not yet finalised by level 1 users within their access region.

C. Enter transport codes and alarm times from patient charts.

D. Contact the responsible dispatch centre to obtain details of the patient (name, date of birth).

E. Twenty-four hours after patient transportation, contact the ICU of the accepting hospital for details of the patient's status, and add these details to the system.

F. Once registration of all details is complete, finalise the case.

Following finalisation of a case, users will have no further access to review the input data.

\section{Level 3 users}

Level 3 users, typically the regional study coordinators, will perform weekly check-ups of data reliability within the system using their personalised username/password combinations. A data set must be authorised by a level 3 user before it can be included in the final database. Unauthorised cases will be stored in a separate database.

The level 3 users will have overviews of complete and incomplete cases. Incomplete cases will be opened and revised by the level 3 user, who will be authorised to add missing information or to overwrite incorrect data. If the registered data have missing values which do not allow calculation of at least the QUIT EMR score, the data set will not be admitted to the final database.

Furthermore, this small group of the highest level of users will be authorised to view all open cases, as well as those which are stored in the complete cases and incomplete cases databases.

After approval of a case for the final database, case identification data will be erased to ensure that the data in the central database are entirely anonymous.

\section{Technical control}

Alongside the groups of medical administrators, continuous technical control and data safety monitoring will be carried out by a technical administrator group from Maastricht University. The work of this group will be independent from that of the medical administrators.

Access to the database will only be possible after authorisation by the coordinating investigator and the technical control staff.

\section{Statistical analysis}

Measured parameters will be represented by mean (SD) or median (IQR) when variables are numerical, and by number $(\%)$ when variables are categorical. All analyses will be performed using IBM SPSS Statistics for Windows. $\mathrm{p}$ Values $\leq 0.05$ will be considered statistically significant. Where appropriate, Independent sample Mann-Whitney $\mathrm{U}$ tests or t-tests will be used to assess changes in the QUIT EMR score, changes in SEMROS or differences in the number of interventions performed between groups of patients who were transported with high or low/medium standard ground transportation systems. Differences in proportion of adverse events between the levels of transportation systems will be tested using $\chi^{2}$ or Fisher's exact test. To account for potential confounders, linear and logistic regression analysis will be performed for numerical and binary outcomes, respectively, in a model including groups of high and low/medium standard ground transportation systems and all baseline variables known to be related to the outcome. Logistic regression analysis will be performed in order to be able to determine which pretransport variable is an independent risk factor for 24 hours post-transportation mortality.

Data mining to identify the impact of other potentially important variables besides type of transportation system shall be performed where appropriate.

\section{Ethics and dissemination}

The study will be conducted in line with the principles of the Declaration of Helsinki amended by the WMA 
General Assembly in October 2013. Only anonymous data from cases of adult patients undergoing physician supervised interhospital transportation will be used for analysis. The study is registered in the Netherlands National Trial Registration: NTR4937. The current data pertaining to the assessment process of both scores are in preparation for publication. Results of this study will determine if a prospective randomised trial involving patients of various categories being randomly assigned to different levels of the transportation system shall be conducted.

\section{DISCUSSION}

Outcomes of this study will be useful for future research, by means of assessing a quality monitoring scoring system and clinically relevant, by taking into consideration the clinical outcomes of patients who were transported with different varieties of vehicles. Therefore, we introduce a clinical score, laboratory value independent.

In the ongoing discussion of centralisation of healthcare facilities, a reliable, efficient and proven safe transport modality which meets the individual patient needs is regarded as the key factor for success in future developments in this field. ${ }^{21-25}$

In clinical practice, the logistic and financial burden of $24 / 7$ provision of transportation facilities remains high. To reduce these pressures, close cooperation within a regional network which prioritises monitoring of quality, such as the cross-border collaboration, the EMR, is necessary.

Simply evaluating the number of critical events or the number of interventions requiring physiological parameter changes during transportation cases cannot provide reliable assessment of the quality of a transportation system. ${ }^{15}$ This is because such events can occur as a result of the natural course of a patient's illness. To overcome this, the QUIT EMR score combines performed interventions of the transportation team with changes in physiological status of the patient. Therefore, events such as a dramatic decrease in blood pressure requiring intervention, which is then adequately treated, would not result in a negative judgement. To the best of our knowledge, such an approach to determine quality of interhospital transportation has yet to be described.

Secondarily, this study examines the possibility of identifying clinically relevant factors which might potentially aid in prediction of 24 hour post-transportation morbidity or mortality.

Currently available and validated scoring systems, such as the SOFA score, require laboratory values which are not always available at the time of transportation. The scoring systems assessed within this study provide a means to calculate the likelihood that a patient's condition will be worse 24 hours after they have been transported, when laboratory values are unavailable.

Moreover, it is expected that sufficient information will be collected to create a new, concrete hypothesis for a randomised controlled non-inferiority trial examining the difference in outcomes of transportation of particular patient categories with either an ICU ambulance or MICU/ITW. This research can be conducted with the use of scoring systems for quality and outcome monitoring. Such research would provide insight into how best transportation resources can be used.

Certainly, the study is not without limitations. Potentially, the greatest limitation is that registration of transport data is voluntary, which may result in a registration bias. Moreover, the registration of transportation data is completed by the concerned transportation team, meaning that no online data are available. In an attempt to create an appropriate compromise between optimising data collected and keeping the registration procedure practical and manageable, it is possible that some aspects of the registration process are unclear, which could result in personal interpretation and discrepancies within collected data. Finally, there is a high logistic burden involved in following up all data; therefore, it might not always be available for all cases.

In conclusion, since it is important that resources are efficiently used, there is a necessity for reliable pretransportation analysis of an individual patient's transportation needs, in combination with standardised quality monitoring. It is hypothesised that outcomes of this study will be able to be used to help create a more evidence-based organisation of interhospital transportation of critically ill patients.

\section{Author affiliations}

${ }^{1}$ Department of Intensive Care, Maastricht University Medical Centre+, Maastricht, The Netherlands

${ }^{2}$ Emergency Medical Service district of Aachen, Aachen, Germany

${ }^{3}$ Emergency Medical Service South Limburg, Geleen, The Netherlands

${ }^{4}$ Department of Methodology and Statistics, Maastricht University, CAPHRI

School for Public Health and Primary Care, Maastricht, The Netherlands

${ }^{5}$ Maastricht University, MEMIC Center for Data and Information Management,

Maastricht, The Netherlands

${ }^{6}$ Emergency Medical Service, Fire Department, Aachen, Germany

Contributors US was coordinating investigator, involved in planning, drafting the original manuscript. DB, JH, JJ, BW, PR participated in study planning, manuscript revision. DV participated in study planning, manuscript revision, and creation of the web application. SB participated in study planning, manuscript revision, providing final approval.

Funding To support the design and construction of the web application, US received a grant from EMRIC+, which is a non-profit international project on cross-border disaster and crisis management.

Competing interests None declared.

Ethics approval Ethics committee of UNiversity Medical Centre Maastricht and UNiversity Hospital of Aachen.

Provenance and peer review Not commissioned; externally peer reviewed.

Data sharing statement The data validation process of two scores is in preparation for publication.

Open Access This is an Open Access article distributed in accordance with the Creative Commons Attribution Non Commercial (CC BY-NC 4.0) license, which permits others to distribute, remix, adapt, build upon this work noncommercially, and license their derivative works on different terms, provided the original work is properly cited and the use is non-commercial. See: http:// creativecommons.org/licenses/by-nc/4.0/ 


\section{REFERENCES}

1. Fanara B, Manzon C, Barbot O, et al. Recommendations for the intra-hospital transport of critically ill patients. Crit Care 2010;14:R87.

2. Lahner D, Nikolic A, Marhofer $P$, et al. Incidence of complications in intrahospital transport of critically ill patients-experience in an Austrian university hospital. Wien Klin Wochenschr 2007;119:412-16.

3. Papson JP, Russell KL, Taylor DM. Unexpected events during the intrahospital transport of critically ill patients. Acad Emerg Med 2007;14:574-7.

4. Beckmann U, Gillies DM, Berenholtz SM, et al. Incidents relating to the intra-hospital transfer of critically ill patients. An analysis of the reports submitted to the Australian Incident Monitoring Study in Intensive Care. Intensive Care Med 2004;30:1579-85.

5. Warren J, Fromm RE, Orr RA, et al. Guidelines for the inter- and intrahospital transport of critically ill patients. Crit Care Med 2004;32:256-62.

6. Ramnarayan P, Thiru K, Parslow RC, et al. Effect of specialist retrieval teams on outcomes in children admitted to paediatric intensive care units in England and Wales: a retrospective cohort study. Lancet 2010;376:698-704.

7. Vos GD, Nissen AC, Nieman FH, et al. Comparison of interhospital pediatric intensive care transport accompanied by a referring specialist or a specialist retrieval team. Intensive Care Med 2004;30:302-8.

8. Bellingan $\mathrm{G}$, Olivier $\mathrm{T}$, Batson $\mathrm{S}$, et al. Comparison of a specialist retrieval team with current United Kingdom practice for the transport of critically ill patients. Intensive Care Med 2000;26:740-4.

9. Wiegersma JS, Droogh JM, Zijstra JG, et al. Quality of interhospital transport of the critically ill: impact of a mobile intensive care unit with a specialized retrieval team. Crit Care 2011;15:R75.

10. Gebremichael M, Borg U, Habashi NM, et al. Interhospital transport of the extremely ill patient: the mobile intensive care unit. Crit Care Med 2000;28:79-85.

11. Strauch U, Bergmans DCJJ, Winkens B, et al. Short-term outcomes and mortality after interhospital intensive care transportation: an observational prospective cohort study of 368 consecutive transports with a mobile intensive care unit. BMJ Open 2015;5:e006801.

12. Barratt $\mathrm{H}$, Harrison DA, Rowan $\mathrm{KM}$, et al. Effect of non-clinical inter-hospital critical care unit to unit transfer of critically ill patients: a propensity-matched cohort analysis. Crit Care 2012;16:R179.
13. Belway D, Henderson W, Keenan SP, et al. Do specialist transport personnel improve hospital outcome in critically ill patients transferred to higher centers? A systematic review. J Crit Care 2006;21:8-17; discussion 17-8.

14. Fan E, MacDonald RD, Adhikari NKJ, et al. Outcomes of interfacility critical care adult patient transport: a systematic review. Crit Care 2006;10:R6.

15. Van Lieshout E, Binnekade J, Reussin E, et al. Nurses versus physician-led interhospital critical care transport: a randomized non-inferiority trial. Intensive Care Med 2016;42:1146-54.

16. Ligtenberg JJM, Arnold LG, Stienstra Y, et al. Quality of interhospital transport of critically ill patients: a prospective audit. Crit Care 2005;9:R446-51.

17. Ramnarayan P. Measuring the performance of an inter-hospital transport service. Arch Dis Child 2009;94:414-16.

18. Uusaro A, Parviainen I, Takala J, et al. Safe long-distance interhospital ground transfer of critically ill patients with acute severe unstable respiratory and circulatory failure. Intensive Care Med 2002;28:1122-5.

19. Seymour CW, Kahn JM, Schwab CW, et al. Adverse events during rotary-wing transport of mechanically ventilated patients: a retrospective cohort study. Crit Care 2008;12:R71.

20. Ferreira FL, Bota DP, Bross A, et al. Serial evaluation of the SOFA score to predict outcome in critically ill patients. JAMA 2001;286:1754-8.

21. Combes A, Luyt CE, Trouillet JL, et al. Adverse effect on a referral intensive care unit's performance of accepting patients transferred from another intensive care unit. Crit Care Med 2005;33:705-10.

22. Durairaj L, Will JG, Torner JC, et al. Prognostic factors for mortality following interhospital transfers to the medical intensive care unit of a tertiary referral center. Crit Care Med 2003;31(7):1981-6.

23. Singh JM, MacDonald RD. Pro/con debate: do the benefits of regionalized critical care delivery outweigh the risks of interfacility patient transport? Crit Care 2009;13:219.

24. Rosenberg AL, Hofer TP, Strachan C, et al. Accepting critically ill transfer patients: adverse effect on a referral center's outcome and benchmark measures. Ann Intern Med 2003;138:882-90.

25. Nguyen YL, Kahn JM, Angus DC. Reorganizing adult critical care delivery. The role of regionalization, telemedicine, and community outreach. Am J Respir Crit Care Med 2010;181:1164-9. 